\title{
A aprendizagem cooperativa e os mapas de conceitos na promoção do pensamento crítico e criativo: uma experiência no ensino superior
}

\author{
Helena Silva, José Lopes \& Caroline Dominguez
}

\begin{abstract}
Resumo:
Este artigo contribui para a reflexão sobre os efeitos do uso da aprendizagem cooperativa e dos mapas de conceitos no desenvolvimento do pensamento crítico e criativo em estudantes do ensino superior. O mesmo apresenta um estudo quasi-experimental, com pré e pós-teste, com um grupo experimental $(n=19)$ e um grupo de controlo $(n=22)$ com estudantes de licenciatura das áreas de Educação Básica e Psicologia. O grupo experimental foi submetido a estratégias de aprendizagem cooperativa e à elaboração de mapas de conceitos a partir da leitura de textos. No grupo de controlo, a metodologia usada na lecionação dos conteúdos foi o método expositivo. As competências de pensamento crítico e criativo de ambos os grupos foram avaliadas com recurso ao Teste de Pensamento Crítico e Criativo (Lopes, Silva, \& Morais, 2019). Os resultados mostraram melhorias significativas nas competências de pensamento crítico e criativo nos alunos do grupo experimental relativamente ao grupo de controlo ( $d=1.66$ ), sugerindo que o uso da aprendizagem cooperativa e dos mapas de conceitos contribuem positivamente para o seu desenvolvimento, especialmente ao nível das competências de explicação e síntese.
\end{abstract}

\section{Palavras-chave:}

aprendizagem cooperativa; pensamento crítico; pensamento criativo; mapas de conceitos; ensino superior. 


\title{
Cooperative learning and concept maps in the promotion of critical and creative thinking: an experience in higher education
}

\begin{abstract}
This article contributes to the reflection on the effects of the use of cooperative learning and concept maps in the development of critical and creative thinking in university students. It presents a quasi-experimental study, with pre and post-test, with an experimental group $(n=19)$ and a control group $(n=22)$ with undergraduate students from the fields of Basic Education and Psychology. The experimental group was submitted to cooperative learning strategies and to the elaboration of concept maps from the reading of texts. In the control group, the teaching methodology used was the traditional lecture. The critical and creative skills of both groups were evaluated using the Critical and Creative Thinking Test (Lopes, Silva, \& Morais, 2019). The results showed significant improvements in critical and creative thinking skills in students in the experimental group compared to the control group $(d=1.66)$, suggesting that the use of cooperative learning and concept maps contributed positively to their development, especially at the level of explanation and synthesis skills.
\end{abstract}

Keywords: cooperative learning; critical thinking; creative thinking; concept maps; higher education.

\section{Apprentissage coopératif et schémas conceptuels dans la promotion de la pensée critique et créative: une expérience dans l'enseignement supérieur}

Résumée: Cet article contribue à la réflexion sur les effets de l'utilisation de l'apprentissage coopératif et des schémas conceptuels sur le développement de la pensée critique et créative chez les étudiants de l'enseignement supérieur. II présente une étude quasi expérimentale, comprenant un groupe expérimental $(n=19)$ et un groupe témoin $(n=22)$ un pré et post-test, avec des étudiants de premier cycle dans les domaines de l'éducation de base et de la psychologie. Le groupe expérimental a été soumis à des stratégies d'apprentissage coopératif et d'utilisation de schémas conceptuels à partir de la lecture de textes. Dans le groupe témoin, la méthodologie d'enseignement utilisée consistait dans l'exposé du professeur. Les compétences de pensée critique et créative des deux groupes ont été évaluées à l'aide du test de pensée critique et créative (Lopes, Silva, et Morais, 2019). Les résultats ont montré des améliorations significatives des capacités de pensée critique et créative chez les étudiants du groupe expérimental par rapport au groupe témoin $(d=1,66)$, ce qui suggère que l'utilisation de l'apprentissage coopératif et des schémas conceptuels ont contribué positivement à leur développement, en particulier au niveau des compétences d'explication et de synthèse.

Mots clés: apprentissage coopératif; pensée critique; pensée créatrive; schémas conceptuels; enseignement supérieur.

\section{El aprendizaje cooperativo y los mapas de conceptos en la promoción del pensamiento crítico y creativo: una experiencia en la enseñanza superior}

Resumen: Este artículo contribuye para la reflexión sobre los efectos del uso del aprendizaje cooperativo y de los mapas de conceptos en el desarrollo del pensamiento crítico y creativo en estudiantes de la enseñanza superior. El mismo presenta un estudio casi-experimental, con pre y post-test, con un grupo experimental $(n=19)$ y un grupo de control $(n=22)$ con estudiantes de licenciatura de las áreas de Educación Básica y Psicología. El grupo experimental fue sometido a estrategias de aprendizaje cooperativo y a la elaboración de mapas de conceptos a partir de la lectura de textos. En el grupo de control, la metodología utilizada en la enseñanza de los contenidos fue el método expositivo. Las competencias de pensamiento crítico y creativo de ambos grupos fueron evaluadas con recurso a la Prueba de Pensamiento Crítico y Creativo (Lopes, Silva, \& Morais, 2019). Los resultados mostraron mejoras significativas en las competencias de pensamiento crítico y creativo en los alumnos del grupo experimental con respecto al grupo de control $(\mathrm{d}=1.66)$, sugiriendo que el uso del aprendizaje cooperativo y de los mapas de conceptos contribuyeron positivamente a su desarrollo, especialmente a nivel de las competencias de explicación y síntesis.

Palabras-clave: aprendizaje cooperativo; pensamiento crítico; pensamiento creativo; mapas de conceptos; enseñanza superior. 


\section{Introdução}

O desenvolvimento do pensamento crítico no ensino superior tem vindo a tornar-se um requisito, não só para o maior sucesso académico dos alunos, mas também para o exercício de uma cidadania plena, na sociedade cada vez mais complexa do século XXI (Pascarella, Wang, Trolian, \& Blaich, 2013). O pensamento crítico é geralmente descrito como uma componente de pensamento de ordem superior que combina várias dimensões: saber analisar, explicar, interpretar, fazer inferências, avaliar, decidir em que acreditar, resolver problemas de forma eficaz, exercitar-se a questionar de forma profunda, ser capaz de detetar fragilidades e aplicar rigor ao seu modo de pensar (autorregulação), entre outras (Paul \& Elder, 2008).

Uma vez que o pensamento crítico tem uma natureza funcional, agregando em si competências relativas ao funcionamento cognitivo ligado à resolução de problemas, não raras vezes se fala de criatividade, ou pensamento criativo, ao falar-se de pensamento crítico. Para Paul e Elder (2008), a criatividade e o pensamento crítico são como os dois lados da mesma moeda. Ou seja, na prática, os dois conceitos estão intrinsecamente ligados desenvolvendo-se em paralelo. O bom pensamento requer a capacidade de gerar produtos intelectuais, o que está associado à criatividade. No entanto, o bom pensamento exige que o indivíduo seja consciente, estratégico e crítico sobre a qualidade desses produtos intelectuais. Como observam os autores referidos, "o pensamento crítico sem criatividade reduz-se ao mero ceticismo e negatividade, e a criatividade sem pensamento crítico reduz-se a mera novidade" (Paul \& Elder, 2008, p. 35).

Apesar da importância do pensamento crítico, surpreendentemente, alguns estudos mostram que o sucesso académico no ensino superior não é sempre acompanhado, como seria expectável, pelo desenvolvimento de competências de pensamento crítico dos estudantes (Lane \& Oswald, 2016). Como qualquer competência, o pensamento crítico e criativo deve ser gradualmente desenvolvido de forma explícita e intencional (Browne \& Keeley, 2001). Duas das estratégias consideradas eficazes na promoção do pensamento crítico são a aprendizagem cooperativa (Loes \& Pascarella, 2017), e o uso dos mapas de conceitos (Novak \& Gowin, 1984).

A aprendizagem cooperativa implica que os alunos trabalhem juntos em grupos pequenos e heterogéneos (3 a 4 elementos) para construir conhecimento e alcançar objetivos de aprendizagem partilhados (Barkley, Cross, \& Major, 2014). Trabalhos anteriores mostram as razões da aprendizagem cooperativa influenciar positivamente o desenvolvimento cognitivo dos alunos (Johnson, Johnson, \& Stanne, 2000). O processo de negociação de significados que acontece nos grupos cooperativos, pelo facto dos estudantes trabalharem juntos para aprender novos conhecimentos e alcançar objetivos partilhados, propicia a ocorrência de conflitos sociocognitivos. Estes mesmos autores argumentam que os alunos em grupos cooperativos partilham conhecimentos e 
reestruturam cognitivamente as informações para as reter na memória e as incorporar nas estruturas cognitivas existentes. Este processo de negociação tem lugar quando os membros do grupo são expostos à heterogeneidade de ideias, à medida que são confrontados com formas inovadoras ou diferentes de analisar problemas familiares (Davidson \& Worsham, 1992).

Em suma, a aprendizagem cooperativa promove o pensamento crítico através da discussão em que os alunos estão envolvidos quando argumentam sobre os diferentes pontos de vista apresentados nos grupos, debatem as suas ideias, sintetizam diferentes informações e avaliam as soluções apresentadas (Klimovienè, Urboniené, \& Barzdžiukiené, 2006). E, comparada com abordagens de tipo mais individualista e expositivo, a aprendizagem cooperativa é considerada como tendo uma influência muito mais significativa no desenvolvimento do pensamento crítico e criativo (Johnson, Johnson, \& Stane, 2000).

Os mapas de conceitos são sistemas de representação gráfica e visual que incluem os conceitos-chave de um tema ou matéria e representam as relações que se estabelecem entre conceitos através de palavras de ligação (proposições) (Novak \& Gowin, 1984). Vários estudos têm demonstrado a importância do uso de mapas de conceitos no desenvolvimento do pensamento crítico (Cañas, Reiska, \& Möllits, 2017). Quando os alunos, individualmente ou em grupo, constroem mapas de conceitos devem integrar todos os conceitos, dos mais gerais aos mais específicos, relacionados com a questão foco e colocar constantemente a si próprios diferentes questões, como por exemplo: "O mapa de conceitos responde à questão foco?"; "Inclui todos os conceitos relacionados com o conceito principal?"; "Estabelece todas as relações entre os conceitos selecionados?". A autorregulação e a heterorregulação que ocorrem quando os alunos questionam, confirmam, verificam ou corrigem raciocínios ou resultados, implicam que mobilizem competências de pensamento crítico como interpretação, análise, avaliação, síntese e façam juízos inferenciais (Cañas, Reiska, \& Möllits, 2017). As ligações cruzadas entre conceitos de diferentes segmentos do mapa implicam também a criação de conhecimento. Este processo representa frequentemente momentos criativos por parte dos alunos como produtores de conhecimento e estes são considerados importantes na facilitação do pensamento criativo (Novak \& Cañas, 2008).

Se a literatura no contexto dos ensinos básico e secundário tem sido abundante em mostrar como a aprendizagem cooperativa influencia positivamente o desenvolvimento cognitivo (Johnson, Johnson, \& Smith, 1991), só mais recentemente esta tem vindo a ser considerada no ensino superior como uma estratégia pedagógica promissora e eficaz (Pascarella \& Teranzini, 2005; Silva et al., 2016). No entanto, são poucas as evidências provenientes de pesquisas experimentais que sustentem a influência positiva da aprendizagem cooperativa no desenvolvimento de competências de pensamento crítico no ensino superior (Notar \& Padgett, 2010). Pelo contrário existem muitas 
investigações que associam a realização de mapas de conceitos ao desenvolvimento de competências de pensamento de ordem superior, em particular ao pensamento crítico (Novak \& Cañas, 2008; Novak \& Gowin, 1984).

Assim, o presente artigo apresenta os resultados de uma investigação que teve como objetivo averiguar em que medida a implementação da aprendizagem cooperativa em conjunto com os mapas de conceitos é benéfica para o desenvolvimento do pensamento crítico e criativo no ensino superior. Mais especificamente, tenta responder à seguinte questão: existe alguma diferença, estatisticamente significativa, entre o uso de uma abordagem que combina a aprendizagem cooperativa e os mapas de conceitos, e o uso de uma abordagem mais tradicional, com recurso ao ensino expositivo, no desenvolvimento de competências de pensamento crítico e criativo de estudantes universitários?

\section{Metodologia}

\subsection{O desenho experimental}

Foi usado um desenho quasi-experimental com um grupo experimental e um grupo de controlo não equivalente (Fraenkel \& Wallen, 2003). A intervenção decorreu durante um semestre letivo, o que correspondeu a 13 aulas de 120 minutos cada. Os alunos do grupo experimental trabalharam em grupos cooperativos de 4 a 5 elementos. Nesses mesmos grupos, os alunos tinham de analisar cenários pedagógicos que envolviam a resolução de problemas e a análise de artigos sobre métodos de ensino e aprendizagem, sobre os quais tinham que igualmente elaborar mapas de conceitos e exercitarem, de forma intencional e explícita, competências de pensamento crítico e criativo. No grupo de controlo, a metodologia usada na lecionação dos conteúdos foi o método expositivo.

\subsection{Participantes}

Participaram 41 estudantes de duas turmas do $3^{\circ}$ ano de uma universidade pública do Norte de Portugal. O grupo de controlo era constituído por 22 estudantes da licenciatura em Psicologia. O grupo experimental era constituído por 19 estudantes da licenciatura em Educação Básica. No que respeita ao género, 87,8\% eram do sexo feminino, com idades entre os 19 e os 37 anos $(M=20,8$; $D P=3,18)$.

\subsection{Instrumentos}

Para avaliar o desenvolvimento do pensamento crítico e criativo, em pré e pós-teste, administrou-se o Teste de Pensamento Crítico e Criativo (TPCC) (Lopes, Silva, \& Morais, 2019), que leva em consideração as taxonomias de Bloom revista (Anderson et al., 2001) e a de Facione (1990). O TPCC apresenta uma situação-problema atual e comum do quotidiano e um conjunto de seis perguntas que requerem a utilização das seguintes competências de pensamento crítico - (i) interpretação, (ii) análise, (iii) 
explicação, (iv) avaliação, (v) síntese - e de pensamento criativo - (vi) produção/criação (fluência, flexibilidade e originalidade).

As respostas dadas às questões são cotadas a partir de uma grelha de avaliação. Para cada pergunta, o avaliador pontua a resposta dada numa escala de 3 ou 4 níveis. Os pontos atribuem-se em função da dimensão do pensamento crítico ou criativo avaliada em cada pergunta, conforme a sua qualidade e quantidade (Tabela 1).

Tabela 1 - Grelha de atribuição de pontos a cada dimensão do pensamento crítico e criativo avaliada.

\begin{tabular}{|c|c|c|}
\hline Dimensão & Critérios de avaliação & Pontos \\
\hline \multirow{4}{*}{$\begin{array}{l}1 . \\
\text { Interpretação }\end{array}$} & Resposta completa, fundamentada e organizada. & 3 \\
\hline & Resposta incompleta, pouco fundamentada e pouco organizada. & 2 \\
\hline & Resposta incompleta, infundamentada e confusa. & 1 \\
\hline & Resposta inteiramente incorreta. & 0 \\
\hline \multirow{4}{*}{ 2. Análise } & $\begin{array}{l}\text { É identificada a totalidade das soluções possíveis, ou ainda, pelo } \\
\text { menos duas diferenças e uma semelhança entre as soluções. }\end{array}$ & 3 \\
\hline & $\begin{array}{l}\text { São identificadas duas soluções possíveis, ou ainda, duas } \\
\text { diferenças, ou uma diferença e uma semelhança, entre as soluções. }\end{array}$ & 2 \\
\hline & $\begin{array}{c}\text { Identifica-se apenas uma solução possível, ou ainda, uma diferença } \\
\text { ou uma semelhança entre as soluções. }\end{array}$ & 1 \\
\hline & $\begin{array}{c}\text { Não se identifica nenhuma solução, ou ainda, não se estabelece } \\
\text { nenhuma comparação entre as soluções. }\end{array}$ & 0 \\
\hline \multirow{4}{*}{ 3. Explicação } & $\begin{array}{l}\text { O argumento é coerente e possui duas ou mais premissas } \\
\text { justificativas. }\end{array}$ & 3 \\
\hline & O argumento é coerente e possui uma premissa justificativa. & 2 \\
\hline & O argumento é pouco coerente. & 1 \\
\hline & O argumento é inválido. & 0 \\
\hline \multirow{4}{*}{ 4. Avaliação } & Resposta coerente e apresenta pelo menos três pontos fracos. & 3 \\
\hline & Resposta coerente e/ou apresenta dois pontos fracos. & 2 \\
\hline & $\begin{array}{l}\text { Resposta pouco coerente e/ou apresenta pelo menos um ponto } \\
\text { fraco. }\end{array}$ & 1 \\
\hline & Resposta apresenta pontos inválidos ou não os apresenta. & 0 \\
\hline \multirow{4}{*}{ 5. Síntese } & $\begin{array}{l}\text { O argumento melhora, de forma coerente, todos os pontos fracos } \\
\text { de uma solução ou origina uma nova solução. }\end{array}$ & 3 \\
\hline & $\begin{array}{l}\text { O argumento melhora, de forma coerente, alguns dos pontos fracos } \\
\text { de uma solução. }\end{array}$ & 2 \\
\hline & $\begin{array}{l}\text { O argumento é pouco coerente com os pontos fracos da solução } \\
\text { em causa. }\end{array}$ & 1 \\
\hline & O argumento não melhora os pontos fracos identificados. & 0 \\
\hline
\end{tabular}




\begin{tabular}{|c|c|c|c|}
\hline \multirow{11}{*}{$\begin{array}{l}\text { 6. Produção/ } \\
\text { Criação }\end{array}$} & \multirow{4}{*}{ Fluência } & Apresenta mais de duas soluções. & 3 \\
\hline & & Apresenta duas soluções. & 2 \\
\hline & & Apresenta uma solução. & 1 \\
\hline & & Não responde. & 0 \\
\hline & \multirow{3}{*}{ Flexibilidade } & $\begin{array}{c}\text { As soluções apresentadas servem para a resolução } \\
\text { do problema. }\end{array}$ & 2 \\
\hline & & $\begin{array}{l}\text { A solução apresentada serve para a resolução do } \\
\text { problema, ou algumas das soluções servem para a } \\
\text { resolução do mesmo. }\end{array}$ & 1 \\
\hline & & $\begin{array}{l}\text { Nenhuma das soluções serve para a resolução do } \\
\text { problema, ou são iguais às propostas no texto. }\end{array}$ & 0 \\
\hline & \multirow{4}{*}{ Originalidade } & $\begin{array}{c}\text { Pelo menos uma das soluções apresentadas é } \\
\text { nova e as outras não têm por base pressupostos } \\
\text { semelhantes às apresentadas, ou ainda, são menos } \\
\text { referidas. }\end{array}$ & 3 \\
\hline & & $\begin{array}{l}\text { Das soluções apresentadas, as que servem para a } \\
\text { resolução do problema são novas e muito referidas. }\end{array}$ & 2 \\
\hline & & $\begin{array}{l}\text { A solução (ou soluções) apresentada(s) é uma } \\
\text { modificação ou melhoria das propostas no texto. }\end{array}$ & 1 \\
\hline & & $\begin{array}{c}\text { A solução (ou soluções) apresentada(s) é uma } \\
\text { cópia das que são propostas. }\end{array}$ & 0 \\
\hline
\end{tabular}

\subsection{Procedimento}

No início do semestre: 1) a docente organizou grupos heterogéneos de quatro e/ou cinco alunos; e 2) foram atribuídos papéis aos diferentes membros do grupo, de forma rotativa e ajustada aos objetivos das atividades. Ao longo das 13 aulas: 3) os alunos no seio dos grupos cooperativos analisaram cenários pedagógicos, que envolviam a resolução de problemas (situações de sala de aula relacionadas com problemas de aprendizagem, indisciplina, falta de motivação) em que os alunos tinham de responder a questões direcionadas para o desenvolvimento das competências de interpretação, análise e síntese); 4) leram e analisaram artigos sobre métodos de ensino-aprendizagem sobre os quais tiveram que desenvolver mapas de conceitos; 5) os trabalhos foram trocados entre os grupos para dar e receber feedback entre pares; 6) a docente deu feedback ao trabalho de cada grupo após o feedback dos colegas; 7) os alunos melhoraram o trabalho realizado, incorporando o feedback dos colegas (feedback entre pares) e o do professor; 8) cada grupo realizou uma apresentação oral final para toda a turma; e 9) cada grupo desenvolveu, periodicamente, uma reflexão sobre o funcionamento do grupo (processo de grupo), nomeadamente relativamente aos pontos fortes, pontos fracos e às possíveis estratégias de melhoria. 
No grupo de controlo, os conteúdos foram apresentados usando uma abordagem mais tradicional - o método expositivo. Tanto o grupo experimental, como o grupo de controlo, responderam ao TPCC na primeira semana de aulas (pré-teste) e na $13^{a}$ semana de aulas (pós-teste).

\subsection{Considerações éticas}

Este estudo seguiu os requisitos éticos da EFPA - Federação Europeia das Associações de Psicólogos, bem como da OPP - Ordem dos Psicólogos Portugueses. Todos os princípios éticos foram respeitados, garantindo que todos os participantes conhecessem e aceitassem os princípios do consentimento informado, a participação voluntária e a confidencialidade das suas respostas.

\section{Resultados}

Todas as análises foram efetuadas com recurso ao programa estatístico SPSS (versão 22.0). Em todas as análises, o nível de significância estatística considerado foi de 5\%.

\subsection{Análise diferencial do pensamento crítico e criativo entre o grupo de controlo} e o grupo experimental em pré-teste

$O$ teste $t$ de amostras emparelhadas revelou diferenças significativas em relação ao pensamento crítico e criativo antes da intervenção $(t(39)=-2,505, p<0,017)$ entre o grupo de controlo $(M=13,68 ; \mathrm{DP}=2,950)$, que tinha uma média mais elevada, e o grupo experimental $(M=11,37$; $D P=2,950)$ (Tabela 2).

Tabela 2 - Análise diferencial dos resultados globais das competências de pensamento crítico e criativo entre os grupos de controlo e experimental em pré e pós-teste.

\begin{tabular}{|c|c|c|c|c|c|c|}
\hline Grupos & $\begin{array}{c}\text { Teste de } \\
\text { Pensamento } \\
\text { Crítico e } \\
\text { Criativo } \\
\text { (TPCC) }\end{array}$ & $\mathrm{N}$ & $\begin{array}{l}\text { Média } \\
\text { (M) }\end{array}$ & $\begin{array}{c}\text { Desvio } \\
\text { Padrão } \\
\text { (DP) }\end{array}$ & $\begin{array}{l}\text { Diferença entre } \\
\text { médias }\end{array}$ & $\begin{array}{c}\text { Magnitude } \\
\text { do efeito } \\
\text { (d de } \\
\text { Cohen) }\end{array}$ \\
\hline \multirow{2}{*}{ Experimental } & Pré-teste & \multirow{2}{*}{19} & 11,37 & 2,95 & \multirow{2}{*}{$t(18)=-6,54, p<.001$} & \multirow{2}{*}{$d=1,66$} \\
\hline & Pós-teste & & 16,53 & 3,27 & & \\
\hline \multirow{2}{*}{ Controlo } & Pré-teste & \multirow{2}{*}{22} & 13,68 & 2,95 & \multirow{2}{*}{$t(21)=1,86, p=0.76$} & \multirow{2}{*}{$d=-0,5$} \\
\hline & Pós-teste & & 12,05 & 3,54 & & \\
\hline
\end{tabular}

\subsection{Análise diferencial do pensamento crítico e criativo entre grupo de controlo} e grupo experimental após a intervenção

Verificou-se um aumento estatisticamente significativo entre as médias do pré-teste $(M=11,37, D P=2,95)$ e as do pós-teste $(M=16,53, D P=3,27, t(18)=-6,54, p$ $<0,001)$ no grupo experimental. Os resultados indicaram uma magnitude de efeito alta 
$(d=1,66)$. Uma análise mais detalhada dos dados mostra que no grupo experimental 84\% dos alunos aumentaram os seus scores no TPCC do pré para o pós-teste (aumento médio de 5,16 pontos). No grupo de controlo, apenas 36,3\% dos estudantes aumentaram os seus resultados do pré para o pós-teste (diminuição média de -1,63 pontos) (Tabela 3).

Tabela 3 - Comparação dos scores médios, desvio-padrão, diferenças de médias, magnitude do efeito no pré-e pós-teste nas competências do pensamento crítico e criativo entre o grupo experimental e do controlo

\begin{tabular}{|c|c|c|c|c|c|c|}
\hline \multirow[b]{2}{*}{ Questão } & \multirow[b]{2}{*}{$\begin{array}{l}\text { Competências } \\
\text { de Pensamento } \\
\text { Crítico e Criativo }\end{array}$} & \multicolumn{5}{|c|}{ Grupo de controlo $(\mathrm{N}=22)$} \\
\hline & & $\begin{array}{c}\text { M } \\
\text { pré- } \\
\text { teste }\end{array}$ & SD & $\begin{array}{c}M \\
\text { pós-teste }\end{array}$ & $\mathrm{SD}$ & $\begin{array}{c}\text { Diferenças } \\
\text { de médias } \\
\text { entre pré e } \\
\text { pós-teste }\end{array}$ \\
\hline 1 & Interpretação & 1,09 & 1,06 & 1,36 & 1 & .27 \\
\hline $2 a$ & Análise & 2,05 & .375 & 2,23 & .42 & .18 \\
\hline $2 b$ & Análise & .86 & .71 & 1,05 & .89 & .19 \\
\hline 3 & Explicação & 1,59 & .66 & 1,81 & .95 & .22 \\
\hline 4 & Avaliação & 1,05 & .66 & .95 & .65 & -.1 \\
\hline 5 & Síntese & 1,23 & 1,11 & 1,64 & 1,32 & .41 \\
\hline 6 & Fluência & 1,45 & .67 & .82 & .5 & -.63 \\
\hline 6 & Flexibilidade & 1,77 & .42 & .77 & .42 & -1 \\
\hline 6 & Originalidade & 2,27 & .55 & 1,55 & .91 & -.72 \\
\hline \multirow[t]{2}{*}{ Pontuação } & TPCC & 13,68 & 2,95 & 12,05 & 3,54 & $-1,63$ \\
\hline & & \multicolumn{5}{|c|}{ Grupo experimental $(\mathrm{N}=19)$} \\
\hline Questão & $\begin{array}{l}\text { Competências } \\
\text { de Pensamento } \\
\text { Critico e Criativo }\end{array}$ & $\begin{array}{c}\text { M } \\
\text { pré- } \\
\text { teste }\end{array}$ & SD & $\begin{array}{c}M \\
\text { pós-teste }\end{array}$ & $\mathrm{SD}$ & $\begin{array}{c}\text { Diferenças } \\
\text { de médias } \\
\text { entre pré e } \\
\text { pós-teste }\end{array}$ \\
\hline 1 & Interpretação & 1,26 & 1,04 & 1,74 & .73 & .48 \\
\hline $2 a$ & Análise & 2,11 & .31 & 2,16 & .5 & .05 \\
\hline $2 b$ & Análise & 1,05 & .4 & 1,63 & .59 & .58 \\
\hline 3 & Explicação & 1,26 & .99 & 2,42 & .96 & 1,16 \\
\hline 4 & Avaliação & .68 & .47 & 1,42 & .69 & .74 \\
\hline 5 & Síntese & 1,32 & 1.1 & 2,47 & .96 & 1,15 \\
\hline 6 & Fluência & 1,53 & .69 & 1,42 & .69 & -.11 \\
\hline 6 & Flexibilidade & .95 & .4 & 1,95 & .78 & 1 \\
\hline 6 & Originalidade & 1,16 & .76 & 1,95 & .8 & .79 \\
\hline Pontuação & TPCC & 11,37 & 2,95 & 16,53 & 3,27 & 5,16 \\
\hline
\end{tabular}


Analisando os resultados obtidos em cada uma das competências em estudo no grupo experimental, verificou-se que, à exceção da fluência, as médias de todas as competências aumentaram do pré para o pós-teste. Os maiores incrementos ocorreram essencialmente nas competências de explicação, síntese, flexibilidade e originalidade (produção/criação). Contrariamente, no grupo de controlo, houve uma diminuição nas médias das competências de avaliação, síntese, fluência, flexibilidade e originalidade. E nas restantes competências, os ganhos foram menores comparativamente aos do grupo experimental.

Neste sentido, os ganhos nas competências de pensamento crítico e criativo no grupo experimental de pré para pós-teste apontam para a diferença das estratégias e metodologias de aprendizagem adotadas durante o semestre.

De uma forma geral, estes resultados parecem indicar que a aprendizagem cooperativa e os mapas de conceitos contribuíram para o desenvolvimento das competências de pensamento crítico e criativo dos alunos. Estes resultados estão de acordo com os estudos de Johnson, Johnson e Stanne (2000) e Klimovienè, Urboniené e Barzdžiukienè (2006), que mostraram que os estudantes melhoram as suas competências de pensamento crítico quando trabalham em grupos cooperativos ao contrário do que acontece quando trabalham individualmente. A aprendizagem cooperativa permitiu que os alunos se envolvessem em processos de interação dialógica, intra e inter grupos, na medida em que tiveram de negociar as suas ideias e pontos de vista (Klimovienè, Urboniené, \& Barzdžiukienè, 2006), o que pode ter levado ao desenvolvimento de competências como a explicação. A aprendizagem cooperativa incentiva a participação ativa na aquisição de conhecimento, promove a interação entre pares, favorecendo o desenvolvimento de competências de reflexão individuais e de grupo relevantes. Quando os alunos têm a oportunidade de interagir com diferentes perspetivas e opiniões sobre o trabalho em questão, como acontece na aprendizagem cooperativa, analisam criticamente as ideias, comentam, comparam o trabalho, dão e recebem feedback o que pode melhorar as suas competências de pensamento crítico (Barnes \& Todd, 1977). De acordo com Beyer (1987), nos grupos cooperativos, os alunos sentem-se livres para arriscar e questionar. $O$ trabalho em grupos cooperativos potencia a interação aluno-aluno focada no processamento de informações, em que os alunos consideram as ideias, contribuições e argumentos dos colegas, comparam-nas com as suas próprias ideias e avaliam a sua pertinência. Os professores não "transmitem" os conteúdos, pelo contrário, incentivam à partilha, ajudam os alunos a analisar criticamente e a negociar as diferentes ideias e opiniões apresentadas para chegarem a consensos. Os alunos são encorajados a tornarem-se aprendizes ativos em vez de recetores passivos de informação; assumem a responsabilidade pelo seu próprio pensamento e aprendizagem (Halpern, 2014). Como Barnes e Todd (1977) afirmam, os adolescentes são capazes de desenvolver competências de pensamento de ordem 
superior (operações formais) através da internalização dos pontos de vista de outras pessoas, o que ocorre durante os diálogos entre pares. O processo de dar sentido ao mundo é profundamente influenciado pelas interações e perceções do ambiente. Tal como Garside (1996) enfatiza, o potencial que os estudantes têm para se elevarem a um nível intelectual de maior desenvolvimento é promovido através da colaboração e da confrontação dos seus pensamentos com os dos outros.

O incremento das competências de análise e essencialmente de síntese poderá dever-se à leitura e análise de artigos científicos para a construção de mapas de conceitos uma vez que, de acordo com Cañas e colegas (2017) a elaboração de mapas de conceitos requer o domínio destas competências. O mesmo pode ter acontecido no que concerne às competências ligadas ao pensamento criativo, com o incremento das competências de flexibilidade e originalidade (Novak \& Canãs, 2008).

Tal como outros estudos empíricos mostraram, quando os alunos se tornam recetivos para considerar outros pontos de vista como válidos, questionam, confirmam, verificam ou corrigem raciocínios ou resultados desenvolvem as suas capacidades de argumentação e contra-argumentação (Loes \& Pascarella, 2017) de interpretação, análise, avaliação e síntese e fazem juízos inferenciais (Novak \& Cañas, 2008), o que foi potenciado pelo trabalho em grupos cooperativos e pelo uso de mapas concetuais.

\section{Conclusões}

Este estudo focou-se na identificação e análise de diferenças estatisticamente significativas entre o desenvolvimento de competências de pensamento crítico e criativo dos estudantes universitários envolvidos numa abordagem de aprendizagem cooperativa usando os mapas concetuais em comparação com aqueles submetidos a um ensino mais tradicional baseado em aulas expositivas presenciais.

Os resultados revelaram que os alunos do grupo experimental, embora partindo de resultados inferiores superaram no pós-teste os resultados do grupo de controlo, tendo assim desenvolvido mais as competências de pensamento crítico e criativo. Estes resultados corroboram estudos recentes que identificam o grande potencial da aprendizagem cooperativa (Devi, Musthafa, \& Gustine, 2015) e dos mapas de conceitos como estratégias para o desenvolvimento do pensamento crítico e criativo (Cañas, Reiska, \& Möllits, 2017).

As competências com aumento mais significativo no grupo experimental, nomeadamente a explicação, a síntese e a flexibilidade (competências de produção/criação), parecem ter sido melhoradas devido às características das atividades desenvolvidas em sala de aula, bem como à participação ativa dos alunos e à interação significativa destes com o material didático. 
As implicações práticas dos resultados deste estudo são claras. Os professores do Ensino Superior serão capazes de aumentar as competências de pensamento crítico e criativo dos alunos ao incluir abordagens de aprendizagem cooperativa, desenhando para o efeito ambientes de aprendizagem propícios para as competências a desenvolver.

Em trabalhos futuros, seria útil investigar o impacto da abordagem de aprendizagem cooperativa ao longo do curso académico, comparando os ganhos em pensamento crítico e criativo entre alunos que aprendem com abordagens cooperativas e outros que aprendem com outras abordagens, bem como avaliar o impacto de variáveis como género, idade e área disciplinar no desenvolvimento dessas competências. Outra importante preocupação de pesquisa deve abordar os efeitos da aprendizagem cooperativa no desenvolvimento de disposições (atitudes como a procura da verdade, curiosidade intelectual, etc.) (Facione, 1990) de pensamento crítico e criativo versus uma abordagem pedagógica mais tradicional.

\section{Referências Bibliográficas}

Anderson, L. W., Krathwohl, D. R., Airasian, P. W., Cruikshank, K. A., Mayer, R. E., Pintrich, P. R., Raths, J., \& Wittrock, M. C. (Org.) (2001). A taxonomy for learning, teaching, and assessing: A revision of Bloom's taxonomy of educational objectives, abridged edition. White Plains, NY: Longman.

Barkley, E. F., Cross, K. P., \& Major, C. H. (Org.) (2014). Collaborative learning techniques: A handbook for college faculty. Hoboken, NJ: John Wiley \& Sons.

Barnes, D., \& Todd, F. (Org.) (1977). Communication and learning in small groups. London: Routledge and Kegan Paul.

Beyer, B. K. (1987). Practical strategies for the teaching of thinking. Boston, MA: Allyn and Bacon.

Browne, M. N., \& Keeley, S. M. (Org.) (2001). Asking the right questions: A guide to critical thinking, Sixth Ed. Upper Saddle River, NJ: Pearson Merrill Prentice Hall.

Cañas, A. J., Reiska, P., \& Möllits, A. (2017). Developing higher-order thinking skills with concept mapping: A case of pedagogic frailty. Knowledge Management \& E-Learning, 9(3), 348-365.

Davidson, N., \& Worsham, T. (Org.) (1992). Enhancing thinking through cooperative learning. New York, NY: Teachers College Press

Devi, A. P., Musthafa, B., \& Gustine, G. G. (2016). Using cooperative learning in teaching critical thinking in reading. English Review: Journal of English Education, 4(1), 1-14.

Facione, P. (1990). Critical thinking: A statement of expert consensus for purposes of educational assessment and instruction. Millbrae, CA: California Academic Press.

Fraenkel, J. R., \& Wallen, N. E. (Org.) (2011). How to design and evaluate research in education. Fifth Ed. New York, NY: McGraw-Hill Education.

Garside, C. (1996). Look who's talking: A comparison of lecture and group discussion teaching strategies in developing critical thinking skills. Communication Education, 45(3), 212-227. 
Halpern, D. F. (2014). Thought and knowledge: An introduction to critical thinking. Fifth Ed. New York, NY: Routledge.

Johnson, D.W., Johnson, R.T., \& Stanne, M.B. (2000). Cooperative learning methods: A meta-analysis. Minneapolis, MN: University of Minnesota.

Johnson, D. W., Johnson, R. T., \& Smith, K. A. (Org.) (1998). Active learning: Cooperation in the college classroom. Edina, MN: Interaction.

Klimovienè, G., Urbonienè, J., \& Barzdžiukienè, R. (2006). Developing critical thinking through cooperative learning. Studies About Languages, 9, 77-85.

Lane, D., \& Oswald, F. L. (2016). Do 45\% of college students lack critical thinking skills? Revisiting a central conclusion of academically adrift. Educational Measurement: Issues and Practice, 35(3), 23-25.

Loes, C. N., \& Pascarella, E. T. (2017). Collaborative Learning and Critical Thinking: Testing the Link. The Journal of Higher Education, 88(5), 726-753.

Lopes, J., Silva, H., \& Morais, E. (2019). Teste do Pensamento Crítico e Criativo para estudantes do ensino superior. Revista Lusófona de Educação, 44(44), 173-189. Disponível em https://revistas. ulusofona.pt/index.php/rleducacao/article/view/6869.

Notar, C. E., \& Padgett, S. (2010). Is think outside the box 21 st century code for imagination, innovation, creativity, critical thinking, intuition? College Student Journal, 44(2), 294-303.

Novak, J. D., \& Cañas, A. J. (Org.) (2008). The theory underlying concept maps and how to construct and use them. Pensacola, FL: Institute for Human and Machine Cognition.

Novak, J. D., \& Gowin, D. B. (Org.) (1984). Learning how to learn. New York, NY: Cambridge University Press.

Pascarella, E. T., \& Terenzini, P. T. (Org.) (2005). How college affects students: A third decade of research (Vol. 2). San Francisco, CA: Jossey-Bass.

Pascarella, E. T., Wang, J. S., Trolian, T. L., \& Blaich, C. (2013). How the instructional and learning environments of liberal arts colleges enhance cognitive development. Higher Education, 66(5), 569583.

Paul, R., \& Elder, L. (Org.) (2008). The miniature guide to critical thinking-concepts and tools, Fifth Ed. Dillon Beach, CA: Foundation for Critical Thinking Press.

Silva, H., Lopes, J., Dominguez, C., Payan-Carreira, R., Morais, E., Nascimento, M., \& Morais, F. (2016). Fostering critical thinking through peer review between cooperative learning groups. Revista Lusófona de Educação, 32, 31-45.

Helena Silva

Escola de Ciências Humanas e Sociais Departamento de Educação e

Psicologia - UTAD

CIIE - Centro de Investigação e Intervenção Educativas

Faculdade de Psicologia e Ciências da Educação - Universidade do Porto

Email: helsilva@utad.pt

ORCID: 0000-0003-2855-9634 
José Lopes

Escola de Ciências Humanas e Sociais Departamento de Educação e

Psicologia - UTAD

CIIE - Centro de Investigação e Intervenção Educativas

Faculdade de Psicologia e Ciências da Educação - Universidade do Porto

Email: jlopes@utad.pt

ORCID: 0000-0002-6845-8371

Caroline Dominguez

Escola de Ciência e Tecnologia Departamento de Engenharias - UTAD LabDCT/CITDFF - Laboratório de Didática de Ciências e Tecnologia/ Centro de Investigação Didática e Tecnologia na Formação de Formadores -

Universidade de Aveiro

Email: carold@utad.pt

ORCID: 0000-0002-8486-4142

Correspondência

Helena Silva

Escola de Ciências Humanas e Sociais

Departamento de Educação e Psicologia

Universidade de Trás-os-Montes e Alto Douro

Quinta de Prados

5000-801-Vila Real, Portugal

Data de submissão: Dezembro 2018

Data de avaliação: Março 2019

Data de publicação: Setembro 2019 